Abstract 419 Table 2 Cox Regression analysis results for mortality risk factors in SLE patients with incident hospital admissions compared to controls.

\begin{tabular}{|c|c|c|c|c|}
\hline \multicolumn{5}{|c|}{ Turianate Cor Regression } \\
\hline & \multirow{2}{*}{ Hazard Ratio } & \multicolumn{2}{|c|}{$5.0 \% \mathrm{CI}$ in $\operatorname{Er}(B)$} & \multirow{2}{*}{ P.ralo } \\
\hline & & Lover & Tper & \\
\hline Lypus Dingoosis & 1656 & 1.47 & 1.895 & $\triangle, 0:$ \\
\hline \multicolumn{5}{|c|}{ Mnltiranite Cos Regression } \\
\hline & \multirow{2}{*}{ Hazard Ratio } & \multicolumn{2}{|c|}{$5.0 \%(I m \operatorname{Inp}(B)$} & \multirow{2}{*}{ P. raloe } \\
\hline & & Lover & Tpper & \\
\hline Lepus Dingaosis & 1991 & 1.473 & 2693 & $ه(0.01$ \\
\hline dge & 1070 & 1060 & 1000 & $\varangle 0.01$ \\
\hline lear of lacident Hospitalsabion & 1047 & 1007 & 1008 & 0.022 \\
\hline Mlales & 1357 & 102.2 & $100 \%$ & 0.035 \\
\hline Length of Stay & 1016 & 1006 & 1006 & $\Delta, 0:$ \\
\hline Thinstred & 1648 & 1389 & 1996 & 0.001 \\
\hline
\end{tabular}

The role of anti-RNP/Sm antibodies in thrombosis deserves further studies

\section{INDONESIAN EPIDEMIOLOGIC DATA OF PAEDIATRIC SYSTEMIC LUPUS ERYTHEMATOSUS}

1,2B Setiabudiawan*, 'R Ghrahani. 'Faculty of Medicine Universitas Padjadjaran, Child Health- Allergy Immunology Division, Bandung, Indonesia; ${ }^{2}$ Chairman of Allergy Immunology, Working Group Indonesian Paediatric Society, Jakarta, Indonesia

\subsection{6/lupus-2017-000215.423}

Background and aims To estimate the epidemiological data of paediatrics systemic lupus erythematosus (SLE) in Indonesia.

Methods A nationwide prospective registry study for the epidemiological data of paediatric SLE was undertaken in Indonesia. Registry data from health service centres in 12 provinces were collected through online registry database since 2012.

Results Two hundred and ten cases of paediatric SLE were identified during the period of 2012-2015. The SLE frequency in girls was 9 times higher than in boys (18:172). The mean age was $11.2 \pm 3.2$ years, with the peak incidence in 13 years old. Most patients were from West Java province, followed by North Sumatra, Jakarta, and South Sulawesi provinces, respectively. The chief complaints were mostly fever, skin disorder, and paleness.
Conclusions This national registry of paediatric SLE in Indonesia provided a good starting point to improve our understanding of the epidemiology of autoimmune diseases in Indonesia. Diagnosis and documentation of this disease are difficult due to challenges in disease recognition and lack of diagnostic facilities; hence, there is a possibility that SLE cases are underdiagnosed in some provinces. Future studies are needed to gain more comprehensive data on nationwide epidemiology of SLE.

424 COULD PRETERM DELIVERY BE A SURROGATE MARKER FOR ACCELERATED DEVELOPMENT OF CARDIOVASCULAR EVENTS IN WOMEN WITH SLE?

1,2,3,4 MC Soh* ${ }^{3} \mathrm{C}$ Nelson-Piercy, ${ }^{1} \mathrm{~L}$ McCowan, ${ }^{5} \mathrm{M}$ Westgren, ${ }^{3} \mathrm{D}$ Pasupathy. ${ }^{1}$ University of Auckland, Department of Obstetrics and Gynaecology, Auckland, New Zealand; ${ }^{2}$ mperial College NHS Trust, Obstetric Medicine Department- Queen Charlotte's and Chelsea Hospital, London, UK; ${ }^{3}$ King's College London, Women's Health Academic Centre, London, UK; ${ }^{4}$ Oxford University Hospitals NHS Foundation Trust, Silver Star High-Risk Pregnancy Unit- John Raddliffe Hospital, Oxford, UK; ${ }^{5}$ Karolinska Insitute, Department of Clinical Science- Intervention and Technology CLINTEC- H9, Stockholm, Sweden 
Abstract 420 Table 1 Patient Characteristics (at index hospitalisation) and study outcomes.

\begin{tabular}{|c|c|c|c|}
\hline & $\begin{array}{c}\text { Lupes vas secoudary } \\
\text { to the Admitting } \\
\text { Dingoosis }\end{array}$ & Watched Cootrols & t2 or titest \\
\hline & $\mathrm{I}_{0} \mathrm{CT}$ or $\mathrm{a}(\mathrm{W})$ & $\mathrm{N} / \mathrm{CT}$ or $\mathrm{a}(\%)$ & (p-rahe) \\
\hline dge & $54.31=18.76$ & $34.32=22.13$ & 0.120 \\
\hline Female & $1911(823 \%)$ & $1735(82.8 \%)$ & 0210 \\
\hline Ladigeoous Status & $8 .(3.5 \%)$ & $26(1.2 \%)$ & $\Delta .0 .01$ \\
\hline Length of Stạ (darys) & $5(2,2,2,11)$ & $?(1 Q R 1,5)$ & $\Delta .001$ \\
\hline Prinately lasured & $438(35.49)$ & $469(48.80 \mathrm{~m})$ & $\triangle 0.0 !$ \\
\hline Diagnosed with a Ischemic Heart Disorder & $184(790 \mathrm{n})$ & $73(2,40)$ & $\Delta .001$ \\
\hline Dizgosed nith a Ceretral Ischemic Disorder & $33(1 .+1 \%)$ & $18(0.8 \%)$ & 0.007 \\
\hline Diagnosed nith a Hipertension Dissorder & $348(15.0 \%)$ & $136(6.90)$ & $\Delta .0 .01$ \\
\hline Diagnosed nith and Atheroscleroto Disorder & $158(6.8 \%)$ & $19(0.900$ & $\$ .001$ \\
\hline Dizgoosed nith a Kitaẹ Disorder & $29(12 \%)$ & $39(1.8 \%)$ & $\Delta .0 .0 !$ \\
\hline Diagnosed rith a Thrombotix Disorder & $34(2.2 \%)$ & $4(02 \%)$ & $\Delta .001$ \\
\hline Pabeat died during the hospital admission & $87(3.7 \%)$ & $40(1.90)$ & $\Delta 0.01$ \\
\hline
\end{tabular}

Background and aims Women with SLE are at greater risk of premature cardiovascular disease and adverse pregnancy outcomes including preterm delivery.

In the general population, preterm delivery is associated with an increased risk of maternal cardiovascular events (CVE).

Therefore, we sought to determine if preterm delivery was a surrogate marker for accelerated CVE in women with SLE. Methods Utilising linked population-based registries from Sweden between 1973-2011, women with SLE born between 1951 - 1971 were included. Preterm delivery was defined as delivery <34 weeks' gestation. Outcome was any CVE (i.e. coronary artery disease, stroke, peripheral vascular disease and death from these causes). Multivariate analysis adjusting for cardiovascular risk factors and SLE-related morbidity (inclusive of inpatient admissions, duration of SLE, renal disease, infection and cancer) was performed.

Results There were 3224 women, median age 49 years (IQR 44-54); 72\% had a previous pregnancy and 6\% had delivered $<34$ weeks' gestation. The prevalence of CVE was $10.4 \%$. Despite being of a similar age distribution, women with $<34$ week deliveries had longer duration of SLE, greater SLErelated morbidity and cardiovascular risk factors.

Those with $<34$ week deliveries had the highest incidence of CVE at the median age of 40.5 years. However, the nonparous group developed CVE earliest.

Conclusions The women with preterm deliveries $<34$ weeks displayed a more severe clinical phenotype of SLE; despite adjusting for these factors, they had an increased hazard of CVE with an accelerated rate of development of CVE 


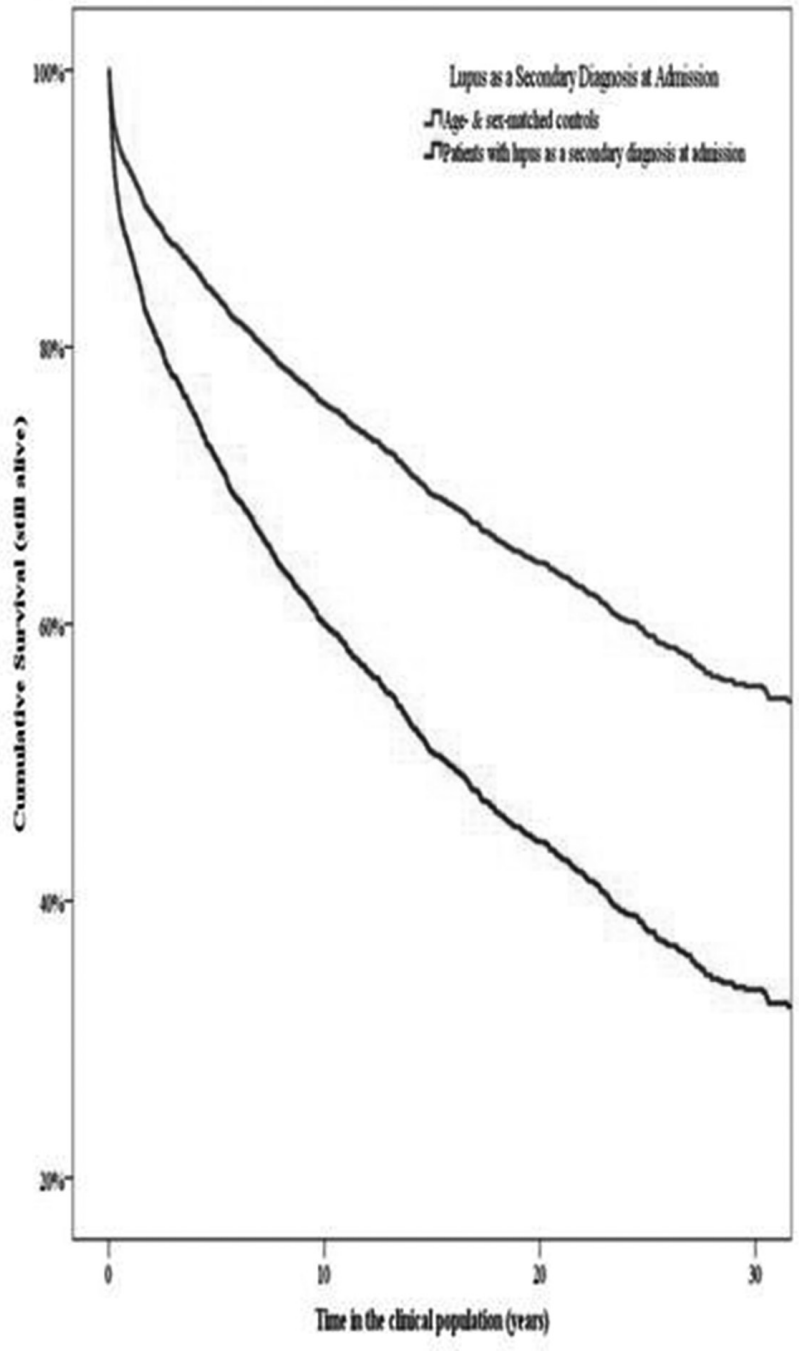

Abstract 420 Figure 1 Kalpan-Meier suvival analysis of mortality outcomes for SLE patients and age- \& sex-matched controls (free of rheumatic disease conditions) from index hospitalisation (Log Rank (Mantel-Cox) $\left.\chi^{2} 158.265, \mathrm{p}<0.001\right)$.

compared to those who delivered later. Preterm delivery could be a surrogate marker for active SLE in pregnancy.

\section{AUTOANTIBODIES PROFILE IN SYSTEMIC LUPUS ERYTHEMATOSUS (SLE) PATIENTS: A STUDY IN MALARIA ENDEMIC AREA}

${ }^{1} \mathrm{R}$ Tripathy*, ${ }^{2} \mathrm{~B}$ Das, ${ }^{2} \mathrm{~S}$ Pattanaik, ${ }^{3} \mathrm{~A}$ Panda. ${ }^{1}$ S. C.B. Medical College, Department of Biochemistry, Cuttack, India; ${ }^{2}$ SCB Medical College, Department of Medicine, Cuttack, India; ${ }^{3}$ Central University of Jharkhand, Centre for Life Sciences, Ranchi, India

\subsection{6/lupus-2017-000215.425}

Background and aims Systemic lupus erythematosus is an autoimmune disorder characterised by elevated levels of autoantibodies. Association of specific autoantibodies with different clinical manifestations of SLE has been documented. Malaria infection is associated with raised levels of autoantibodies. It is not known what happens to autoantibody productions in SLE patients residing in malarial endemic areas and its association with disease manifestation. In the present study,we enrolled SLE patients residing in from different areas of Odisha state, Indiaand investigate possible association of $P$. falciparum endemicity with autoantibodies profile of SLEpatients.

Methods A total of 190 SLE patients from different districts of Odisha, which is endemic for $P$. falciparum malaria were enrolled in the present study. Clinically assessed, and autoantibodies levels were quantified by standard laboratory procedures. They were grouped as 1) Patients from low endemic area based on Annual Parasite Index (API) as low (API <4) and 2) High (API >4) and the data was analysed.

Results The mean age and disease duration of SLE patients were 28.44 and 1.67 years, respectively. 128 patients resided in lower endemic areas and 62 in high endemic areas. 62\%of patients from higher endemic areas had nephritis and other major manifestations like NPSLE and myocarditis. Interestingly, SLE patients from areas(API >4) displayed higher levels of anti-Sm. Other auto antibodies levels were comparable among the two groups.

Conclusions The results of the present study revealed an association of malarial endemicity with differential production of autoantibodies, namely Sm. However, the role of malaria in the pathogenesis of SLE needs to be validated in a prospective study.

\section{RENAL ACTIVITY AND DAMAGE INCUR HIGHEST MEDICAL COSTS AMONG FILIPINO PATIENTS WITH SYSTEMIC LUPUS ERYTHEMATOSUS}

E Venegas*, K Geslani, S Navarra. University of Santo Tomas Hospital, Rheumatology, Manila, Philippines

\subsection{6/lupus-2017-000215.426}

Background and aims Systemic lupus erythematosus (SLE) predominantly affects individuals at peak age of productivity, and medical costs negatively impact on personal, family and community resources. This study aimed to identify annual medical costs and cost predictors among Filipino SLE patients.

Methods Direct annual healthcare costs were determined by survey questionnaires conducted among patients aged $>18$ years with minimum 1 year illness duration, consecutively seen at Lupus Clinics of University of Santo Tomas (UST) Hospital, Manila, Philippines from February to July 2016. Excluded were costs related to biologics. Predictors of cost were estimated using multiple regression analysis.

Results Respondents included 300 SLE patients (93.7\% female) with mean age $32.84 \pm 9.89$ (11-62) at diagnosis, mean disease duration $5.87 \pm 5.58$ (1-36) years. Median and mean annual direct medical cost was Php 90950 and Php 133040 respectively (range Php 17 440-859,050). Annual cost was higher in those requiring dialysis $(n=16)$ compared to nephritis without dialysis $(n=150)$ vs without nephritis ever $(n=134)$, (median Php 595400 vs 144700 vs 55020$), p<0.001$. End stage renal disease (ESRD) $(\mathrm{p}<0.001)$, mycophenolate use $(\mathrm{p}<0.001)$, clinic visits $(\mathrm{p}=0.016)$ and hospitalisation $(p=0.018)$ were independent variables which significantly contributed to annual costs. [Php47.5 = USD1]

Conclusions Nephritis especially if requiring dialysis was the most important cost predictor in this cohort, increasing annual costs up to 7 times. Mycophenolate use, frequency of hospitalisation and clinic visits increased annual costs by $147.2 \%$, $173.8 \%$ and $2.6 \%$ respectively. This study reinforces need for 